\title{
Cytosine-based nucleoside analogs are selectively lethal to DNA mismatch repair- deficient tumour cells by enhancing levels of intracellular oxidative stress
}

\author{
M Hewish ${ }^{1,2,3}$, S A Martin ${ }^{1,2,4}$, R Elliott ${ }^{1}$, D Cunningham ${ }^{3}$, C J Lord ${ }^{\star, 1}$ and A Ashworth ${ }^{\star, 1,2}$ \\ ${ }^{1}$ The Breakthrough Breast Cancer Research Centre, The Institute of Cancer Research, Fulham Road, London, SW3 6JB, UK; ${ }^{2} C R U K$ \\ Gene Function Laboratory, The Institute of Cancer Research, Fulham Road, London SW3 6JB, UK and ${ }^{3}$ Department of Medicine, \\ Royal Marsden Hospital NHS Trust, London and Surrey, UK
}

Background: DNA mismatch repair deficiency is present in a significant proportion of a number of solid tumours and is associated with distinct clinical behaviour.

Methods: To identify the therapeutic agents that might show selectivity for mismatch repair-deficient tumour cells, we screened a pair of isogenic MLH1-deficient and MLH1-proficient tumour cell lines with a library of clinically used drugs. To test the generality of hits in the screen, selective agents were retested in cells deficient in the MSH2 mismatch repair gene.

Results: We identified cytarabine and other related cytosine-based nucleoside analogues as being selectively toxic to MLH1 and MSH2-deficient tumour cells. The selective cytotoxicity we observed was likely caused by increased levels of cellular oxidative stress, as it could be abrogated by antioxidants.

Conclusion: We propose that cytarabine-based chemotherapy regimens may represent a tumour-selective treatment strategy for mismatch repair-deficient cancers.

Mismatch repair deficiency (dMMR) is recognised as an important cause of mutagenesis and driver of oncogenesis in human cancer (Jiricny, 2006; Hewish et al, 2010). Mismatch repair deficiency is manifest in a small proportion of solid tumours that occur in association with the inheritance of a pathogenic mutation in an MMR gene (Lynch syndrome) (Lynch et al, 2008), but is also frequently involved in sporadic tumorigenesis. Fifteen per cent of all colorectal cancers (CRC) exhibit dMMR, with the majority occurring as a consequence of hypermethylation of the MLH1 gene promoter (Hewish et al, 2010; Tejpar et al, 2010). More recently, with access to high-throughput sequencing techniques, sporadically occurring mutations in MMR genes have been reported in cancers not classically associated with Lynch syndrome (Maeng et al, 2012). Given the widespread occurrence of dMMR and the availability of robust biomarkers to determine the presence of dMMR in human tissue (Hewish et al, 2010), we sought to identify new therapeutic strategies for this subgroup of cancers.

The classical MMR pathway primarily exists to repair base-base mismatches and short insertion-deletion loop mismatches that form during DNA replication (Jiricny, 2006). Canonical MMR involves two protein containing heterodimers, MutS and MutL. The MSH2-containing heterodimer (MutS) senses the mismatch and triggers the recruitment and activation of the MLH1containing heterodimer (MutL) that in turn coordinates other proteins, which sequentially excise, resynthesise, and religate the repaired DNA at the mismatched site (Jiricny, 2006; Hewish et al,

\footnotetext{
*Correspondence: Dr CJ Lord; E-mail: Chris.Lord@icr.ac.uk or Professor A Ashworth; E-mail: Alan.Ashworth@icr.ac.uk

${ }^{4}$ Present address: Centre for Molecular Oncology, Barts Cancer Institute, Queen Mary University of London, Charterhouse Square, London, EC1M 6BO, UK
} 
2010). In the absence of functional MMR, for example, caused by MSH2 or MLH1 mutation, this process becomes dysregulated and can lead to a 'mutator phenotype', with the rapid accumulation of thousands of mutations throughout the genome, including those in microsatellite repeat regions. The majority of dMMR tumours, including over $90 \%$ of those deficient in the commonest MMR defects, MLH1 and MSH2, exhibit microsatellite instability (MSI) (Hewish et al, 2010). In addition to their role in the repair of DNA lesions during DNA replication, it is increasingly appreciated that MMR proteins also play important roles in other processes, including the repair of oxidatively damaged DNA (Macpherson et $a l, 2005)$. These additional functions are thought to be responsible for the altered responses of dMMR cancer cells to cytotoxic chemotherapy, including the relative resistance of $\mathrm{dMMR}$ CRC cells to 5-fluorouracil (Hewish et al, 2010).

MMR genes function as classical tumour suppressor genes, with loss of function of both alleles being generally required to cause the dMMR phenotype (Hewish et al, 2010). As therapeutic restoration of normal gene function is complex, synthetic lethal strategies have been proposed as a means of targeting cancers driven by tumour suppressor gene dysfunction (Ashworth et al, 2011). Synthetic lethality describes the relationship between two genes or pathways, where loss of function of one gene or pathway in a cell is compatible with cellular viability, whereas loss of both is not. In part, the promise of synthetic lethality has been borne out by the successful translation of the sensitivity of tumours deficient in BRCA1 or BRCA2 to treatment with poly (ADPribose) polymerase (PARP) inhibitors (Tutt et al, 2010; Ashworth et al, 2011). Synthetic lethal approaches to dMMR have also been proposed, including inhibition of PINK1 as being selectively cytotoxic to MMRdeficient cells (Martin et al, 2011) and exposure to methotrexate as being selectively cytotoxic to MSH2-deficient cells (Martin et al, 2009). Here, we describe a comprehensive drug screen to identify additional synthetic lethal targets for $\mathrm{dMMR}$ and in doing so highlight the potential for cytosine-based nucleoside analogues as a potential therapeutic strategy.

\section{MATERIALS AND METHODS}

Cell lines, culture conditions, and reagents. HCT116 and HCT116 + Chr3 were a gift from Dr A Clark, National Institute of Environmental Health Sciences, Research Triangle Park, NC, USA. BVEC F7bro and BVEC E2 are HCT116 derivatives and were donated by Professor Bert Vogelstein, Johns Hopkins University of Medicine, Baltimore, MD, USA. LoVo (and LoVo + Chr2 cells, created by Dr Minoru Koi) were donated by Professor Rick Boland, Baylor University Medical Centre, Dallas, TX, USA and Dr Christopher Gasche, Medical University of Vienna, Austria. A2780cp70 a2 and A2780cp70 e1 cells were donated by Professor Robert Brown, Institute of Cancer Research, Surrey, UK. SW48, CACO2, HCT-15, RKO, HRT-18, and SW480 were obtained from the ATCC, and HT29, LS174T, COLO205 and COLO-741 from the ECACC. Cells were cultured in growth media supplemented with $10 \%$ Fetal bovine serum (Gibco, Life Technologies, Paisley, UK), $2 \mathrm{~mm}$ L-glutamine (Gibco), $50 \mathrm{U} \mathrm{ml}^{-1}$ penicillin and $50 \mu \mathrm{g} \mathrm{ml}^{-1}$ in a $5 \%$ $\mathrm{CO}_{2}$ environment at $37^{\circ} \mathrm{C}$. HCT116 and derivatives, and HRT-18 were maintained in McCoy's 5A media (Gibco), with the addition of the selection pressure of $400 \mu \mathrm{g} \mathrm{ml}^{-1}$ geneticin to HCT116+Chr3. LoVo were maintained in Iscove's modified Dulbecco's medium, with the addition of $700 \mu \mathrm{g} \mathrm{ml}^{-1}$ geneticin to $\mathrm{LoVo}+\mathrm{Chr} 2$. A2780cp70, RKO, COLO205 and COLO-741 were maintained in Roswell Park Memorial Institute (RPMI) media (Gibco), with the addition of $200 \mu \mathrm{g} \mathrm{ml}^{-1}$ hygromycin B to A2780cp70 a2 and A2780cp70 e1. LS174T, HCT-15, SW48, SW480, CACO2, and HT29 were maintained in Dulbecco's modified Eagle's medium (Gibco). Cells were expanded for two passages and then cryopreserved.
Cytarabine (Ara-C), azacytidine, cyclocytidine, and nucleoside triphosphates (Sigma Aldrich, Dorset, UK) were dissolved in phosphate-buffered saline (PBS). N-acetyl-l-cysteine (Sigma) was dissolved in media containing HEPES (4-(2-hydroxyethyl)-1piperazineethanesulfonic acid) buffer, adjusting $\mathrm{pH}$ using $1 \mathrm{M}$ $\mathrm{NaOH} .{ }^{3} \mathrm{H}$-cytarabine was obtained from Moravek Biochemicals (Brea, CA, USA). Drugs were stored at $-20^{\circ} \mathrm{C}$.

Drug screening and validation. The contents of the commercially available US Drugs library (http://www.msdiscovery.com) were dissolved in DMSO and stored at $-20{ }^{\circ} \mathrm{C}$. For all cell viability experiments, cells were seeded in 96 or 384-well plates and exposed to serial dilutions of drug or DMSO/PBS control $24 \mathrm{~h}$ later. Cell viability was assessed at the end of the experiment using the CellTiter-Glo luminescent assay (Promega UK, Southampton, UK) according to the manufacturer's instructions with an automated plate reader. Luminescence readings for wells exposed to control were compared with wells exposed to test drug in order to calculate a surviving fraction (SF). Data were analysed using GraphPad Prism (GraphPad Software Inc., La Jolla, CA, USA) and survival curves determined using the Four Parameter Logistic Regression Model. For clonogenic assays, cells were seeded in six-well plates and exposed to serial dilutions of drug of DMSO/PBS control. Drug or media was replaced twice a week till the end of the experiment (10-14 days). Colonies were washed in PBS, fixed in methanol, stained using crystal violet solution, washed and colonies counted using an automated colony counter (Oxford Optronics, Oxford, UK), and SFs calculated.

Short interfering RNA transfections. Cells were reverse transfected with short interfering RNA (siRNA) duplexes targeting MLH1, POLA, POLA2, PLK1 or non-targeting control (Supplementary Methods). Twenty-four hours after transfection, cells were trypsinised, counted, and reseeded in 96-well plate format, exposed to drug or control $24 \mathrm{~h}$ later, and viability assessed.

Protein analysis. Whole-cell extracts were prepared by lysing cells in NP-40 lysis buffer with protease inhibitors (Complete mini protease inhibitor cocktail tablets, Roche, Welwyn Garden City, UK). Lysates were electrophoresed on NuPAGE Tris-acetate precast gels (Invitrogen, Life Technologies), and immunoblotted with the following antibodies: anti-PARP (Santa Cruz, Sc-8007, 1: 1000, Santa Cruz Biotechnology, Santa Cruz, CA, USA), anti-MLH1 (Calbiochem PC56 ab-2, 1:1000, Calbiochem, Nottingham, UK), and anti-Phospho (Ser139) H2AX (Upstate 05-636, 1:1000). Immunoblotting for anti- $\beta$-actin-HRP (Santa Cruz Sc-1616, $1: 250$ ) was used as a loading control. This was followed by incubation with anti-IgG-horseradish peroxidase and chemiluminescent detection (Supersignal West Pico chemiluminescent substrate) (Pierce, Thermofisher Scientific, Rockwell, IL, USA).

Immunofluorescence. Cells grown on coverslips were washed and fixed with $4 \%$ paraformaldehyde. Cells were permeabilised in $0.2 \%$ $(\mathrm{v} / \mathrm{v})$ Triton X-100 in PBS for $30 \mathrm{~min}$, washed, blocked and incubated with anti-Phospho (Ser139) H2AX antibody (Cell Signaling 9718 S, 1:1000, Cell Signaling, New England Biolabs, Hitchin, UK), followed by an Alexa Fluor $555 \mathrm{~nm}$ (Molecular Probes, Invitrogen, Life Technologies)-conjugated secondary antibody diluted $1: 1000$. Coverslips were washed in DAPI $\left(4^{\prime}, 6-\right.$ diamidino-2-phenylindole) diluted $1: 10000$ in PBS (358 nm excitation, Molecular Probes) to visualise cell nuclei, placed on Vectashield mounting medium (Vectorlabs, Peterborough, UK) on a microscope slide, and viewed on a Leica TCS-SP2 confocal microscope (Leica Microsystems, Milton Keynes, UK).

Analysis of cytarabine incorporation into DNA. Cells were treated with $50 \mathrm{~nm}$ cytarabine mixed with $20 \mu \mathrm{Ci}$ of ${ }^{3} \mathrm{H}$-cytarabine. DNA from cytarabine-treated cells and controls was purified using the QIAamp DNA Blood Mini Kit (Qiagen, Manchester, UK). 

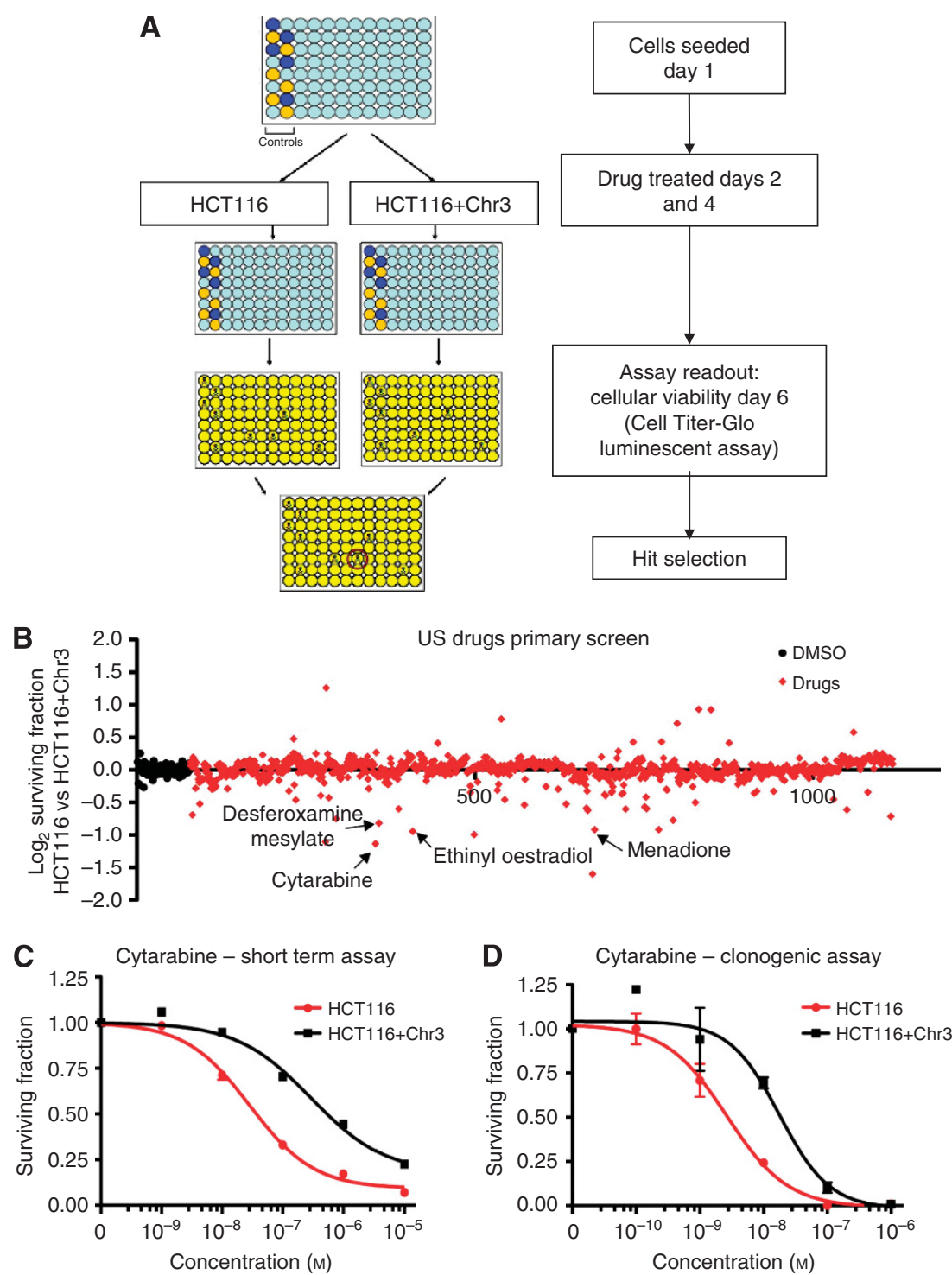

Figure 1. Primary screen of MLH1-deficient and proficient cancer cells identifies cytarabine as MLH1-deficient selective. (A) The MLH1-deficient CRC cell line HCT116 and the MLH1-proficient comparator HCT116+Chr3 were screened in parallel. Cells were plated on day 1 and exposed to drug or control continuously from day 2. Viability was assessed using a luminescent assay on day 6. Data were analysed to obtain MLH1-deficient selective hits. (B) Scatter plot of the primary screen. $\log _{2}$ SF for HCT116 was compared with the $\log _{2}$ SF for HCT116 + Chr3 and plotted against screen position. (C, D) Survival curves from assays of the effects of cytarabine on (C) cell viability in HCT116+/ - Chr3 cells exposed continuously to drug and (D) clonogenic survival in HCT116 $+/-$ Chr3 cells exposed to $24 \mathrm{~h}$ of cytarabine. Assays were performed in triplicate or quadruplicate. Error bars represent the standard error of the mean (s.e.m.).

DNA samples were quantified and counts per minute measured on a 2500 TR liquid scintillation counterPackard. The incorporation of ${ }^{3} \mathrm{H}$-cytarabine in each sample was calculated from the activity of ${ }^{3} \mathrm{H}$-cytarabine recorded expressed as DPM/l (disintegrations per minute), compared with total DNA in the sample analysed.

Analysis of 8-oxo-7,8-dihydro-2'-deoxyguanosine (8-oxodG). Genomic, nuclear, and mitochondrial DNA were extracted from cell lines as previously described (Martin et al, 2011). Following quantification, DNA samples were standardised, digested with nuclease P1 (Sigma), treated with calf intestinal phosphatase and denatured. 8-oxo-7,8-dihydro- $2^{\prime}$-deoxyguanosine (8-oxodG) levels were quantified using an 8-oxodG ELISA assay in 96-well plate format (Cayman Chemicals, Ann Arbor, MI, USA) following the manufacturer's instructions. Standard curves were calculated from serial dilutions of 8-oxodG standard to calculate reaction efficiency. Absorbance was quantified at $405 \mathrm{~nm}$ using an automated plate reader.
Analysis of reactive oxygen species. Reactive oxygen species (ROS) and reactive nitrogen species (RNS) in cellular samples and culture supernatant samples were assessed using the OxiSelect In Vitro ROS/RNS Assay Kit (Cell Biolabs, Inc, San Diego, CA, USA), according to the manufacturer's instructions. Cells and supernatant samples were homogenised and particulate matter removed. Samples were assayed for the presence of fluorescent dichlorodihydrofluorescein (DCF), produced on the oxidation of DCFHDioOxyQ (DCF DiOxyQ), and compared against a standard curve. Fluorescence was quantified using an automated plate reader, and normalised to cell number.

\section{RESULTS}

Isogenic MMR-deficient and proficient drug screens. To model the effects of MLH1 deficiency in vitro, we used the human CRC cell line HCT116 and its isogenic comparator HCT116+ Chr3. 
A

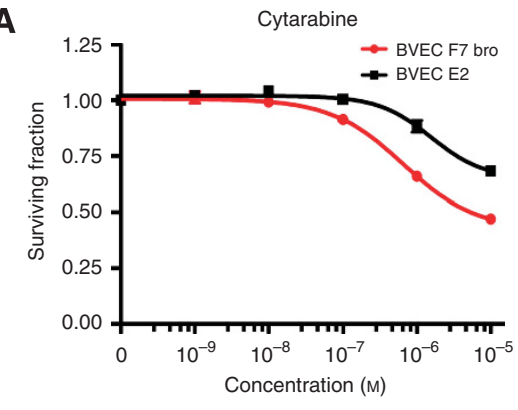

C
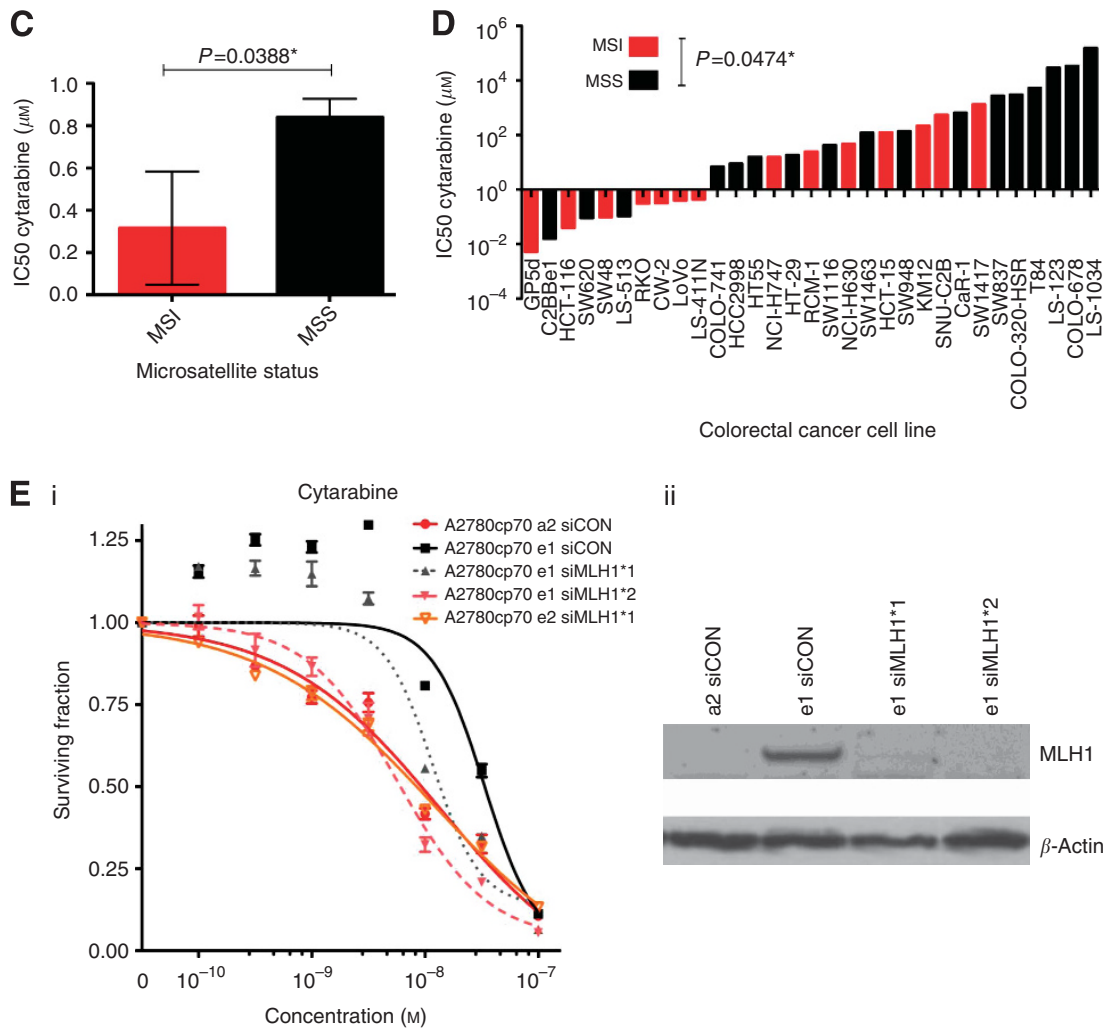

Colorectal cancer cell line

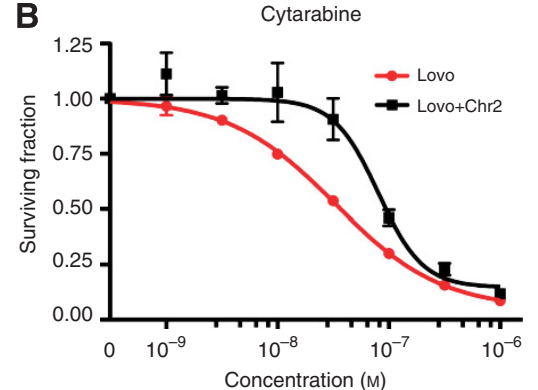

$\mathrm{ii}$

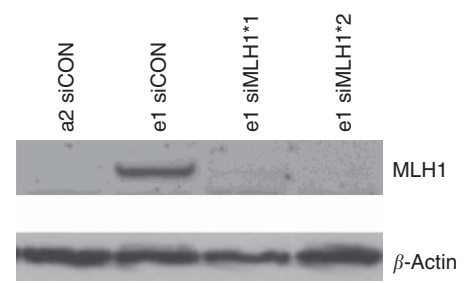

Figure 2. Multiple models of MMR deficiency are selectively sensitive to cytarabine. (A) Cell viability assay of BVEC F7bro and E2 cells exposed to cytarabine for $24 \mathrm{~h}(P \leqslant 0.0001$ using ANOVA). (B) Cell viability assay of LoVo and LoVo + Chr2 cells, following continuous exposure to cytarabine. (C) Box and whisker plot of cytarabine IC50 values obtained from the Sanger Institute data set, stratified by MSI status. $(P=0.0388$ using two-tailed t-test). (D) Waterfall plot of cytarabine IC50 values in CRC cell lines obtained from the same data set, stratified by MSI status $(P=0.0474$ using two-tailed t-test). (E(i)) Survival curves from cell viability assays of A2780cp70 a2 and e1 cells transfected with non-targeting control siRNA or siRNA targeting MLH1, and continuously exposed to various concentrations of cytarabine, vs control, $48 \mathrm{~h}$ after transfection. Assays were performed in triplicate or quadruplicate. Error bars represent the s.e.m. (E(ii)) Immunoblot of MLH1 expression in cell lysates obtained from this experiment, with $\beta$-actin as loading control.

HCT116 is MLH1-deficient because of the presence of homozygous mutations in exon 9 of the MLH1 gene, while HCT116+ Chr3 has been rendered MLH1-proficient due to the stable transfer of a copy of chromosome 3 (and a wild-type $M L H 1$ gene) using microcell fusion (Koi et al, 1994). To identify potential drugs that selectively target MLH1-deficient cells, we screened both cell lines with the contents of the commercially available US Drug Collection, containing 1040 drugs that have entered clinical assessment.

The drug library contents were arrayed in 96-well plate format, with control wells in each plate. Cell solutions were dispensed using an automated liquid handling device, and cells exposed to drug (at a concentration of $10 \mu \mathrm{M})$ or control $(0.1 \%$ DMSO) in growth media for 5 days, $24 \mathrm{~h}$ after seeding (Figure 1A). Cell viability was estimated at the end of the experiment using the luciferase-based CellTiter-Glo assay Promega, which quantifies ATP. The effect of each compound was assessed by comparing log- transformed average readings from control wells with drugtreated wells on that plate, to obtain a $\log _{2}$ SF. The $\log _{2}$ SFs for each cell line were compared with select MLH1-deficient selective drugs. The screen was performed twice and results combined in the final analysis.

A scatter plot comparing the $\log _{2}$ ratio of SFs obtained in the MLH1-deficient and MLH1-proficient cell lines is shown in Figure 1B. Drugs demonstrating MLH1-deficient selectivity are listed in Supplementary Table 1. Among drugs identified as being MLH1-deficient selective were menadione, ethinyl estradiol, and desferrioxamine, which have been associated previously with modulation of cellular oxidative stress (for example (Laux and $\mathrm{Nel}, 2001)$ ), consistent with our previous findings (Martin et al, $2009,2011)$. While menadione has been previously validated as being MSH2-deficient selective (Martin et al, 2009), it has been withdrawn from clinical use. Nevertheless, the identification of 
A

\begin{tabular}{|c|c|c|c|c|c|c|c|}
\hline \multicolumn{3}{|c|}{ HCT116 } & & \multicolumn{3}{c|}{ HCT116+Chr3 } & Treatment \\
\hline- & + & - & & - & + & - & Ara-C 50 nM \\
\hline- & - & + & & - & - & + & Ara-C 100 nM \\
\hline
\end{tabular}
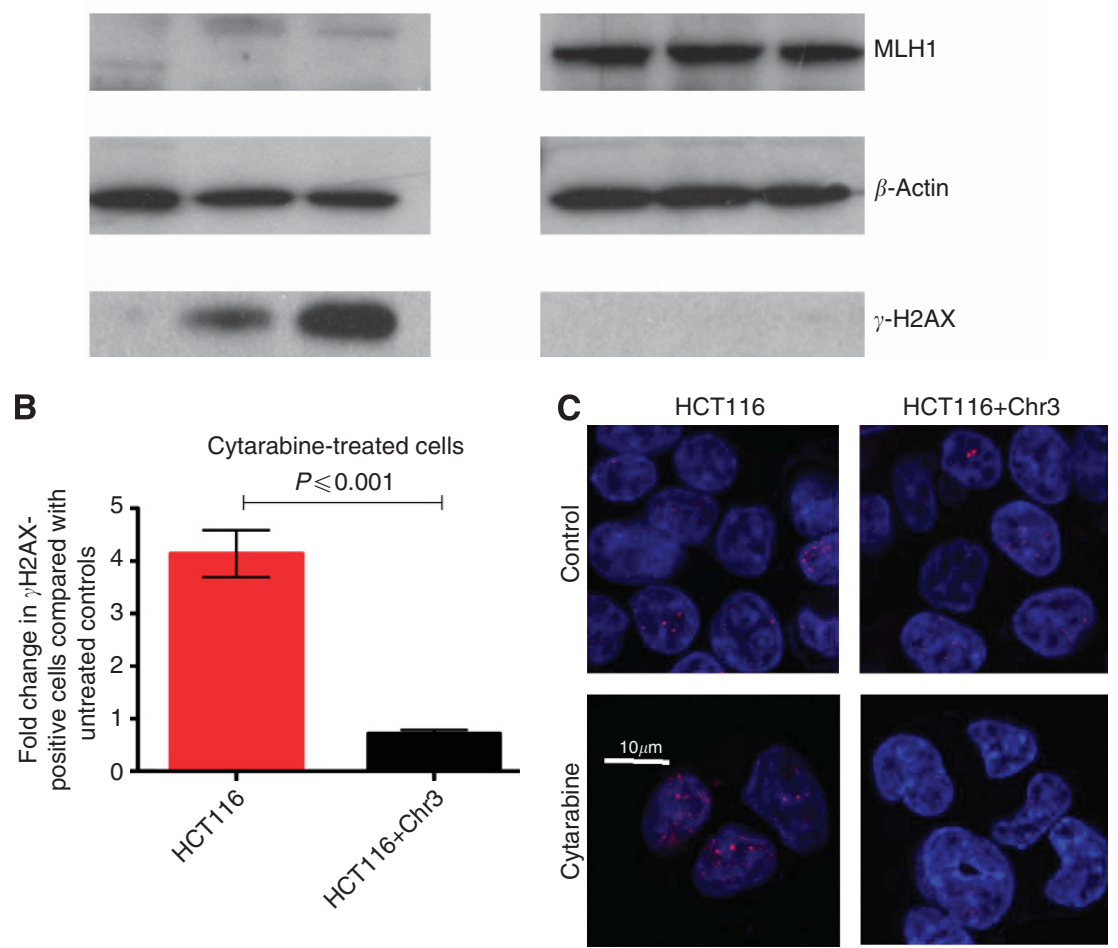

Figure 3. The excess cytotoxicity of cytarabine in MMR-deficient cells is associated an increase in markers of apoptosis and DNA damage. (A) Immunoblot assessing the effects of cytarabine treatment on protein levels. Lysates were obtained from HCT116 $+/-$ Chr3 cells exposed to cytarabine (ara-C), or control, for $72 \mathrm{~h}$. Lysates were immunoblotted and probed for expression of PARP, MLH1, $\gamma \mathrm{H} 2 \mathrm{AX}$, and $\beta$-actin as a loading control. (B) Bar graph demonstrating percentage change in $\gamma \mathrm{H} 2 \mathrm{AX}$-positive cells by confocal microscopy following treatment with $50 \mathrm{~nm}$ cytarabine for $24 \mathrm{~h}$, compared with controls. Cells were fixed $24 \mathrm{~h}$ following cytarabine exposure. $\geqslant 5$ foci per cell was defined as a positive cell. At least 100 cells were counted in a minimum of seven representative fields. (C) Merged images of control (PBS-treated) HCT116 and HCT116 + Chr3 cells (top panels) and cytarabine-treated cells (bottom panels). Cells were stained for $\gamma \mathrm{H} 2 \mathrm{AX}$ (red) and DAPI (blue).

menadione demonstrated that we could identify MMR selective agents from this screen.

Cytarabine is selective for MLH1-deficient cells. We noted that the nucleoside analogue, cytarabine was selective for MLH1deficient HCT116 cells in the screen with a significant difference in SFs (SF at $10 \mu \mathrm{M}$ cytarabine for HCT116 $=0.0891$ vs HCT116 + $\mathrm{Chr} 3 \mathrm{SF}=0.3289 ; \log _{2}$ ratio -1.134 ) (Supplementary Table 1$)$. As screens are prone to a significant false-positive rate, we assessed the effects of cytarabine using short-term viability assays (with identical conditions to the original screen) (Figure 1C), and clonogenic assays, the gold standard of cellular viability (Brown and Attardi, 2005), where cells were exposed to cytarabine for $24 \mathrm{~h}$ (Figure 1D). We observed that cytarabine was MLH1-deficient selective in both assays, and that the magnitude of MLH1-deficient selective cytotoxicity was increased with increased length of drug exposure (Supplementary Figure 1), suggesting that the observed differential phenotype was perhaps due to a cumulative effect.

A synthetic lethal interaction that is relatively unaffected by other genetic changes has been termed a 'hard' synthetic lethality (Ashworth et al, 2011). To determine whether our observations were generally applicable to models of $\mathrm{dMMR}$, we assessed the effect of cytarabine in other isogenic MLH1 and MSH2-deficient cell line pairs. We used the CRC cell lines, BVEC F7bro (MLH1deficient) and BVEC E2 (MLH1-proficient) (Weiss et al, 2007), as well as LoVo (MSH2-deficient) and $\mathrm{LoVo}+\mathrm{Chr} 2$ (MSH2 proficient) (Watanabe et al, 2000). We found that cytarabine was selective for MMR-deficient cell lines in both systems (Figures 2A and $\mathrm{B}$ ), suggesting that this phenotype was applicable to multiple models.

To determine the generality of our observations in a nonisogenic setting, we interrogated the publicly available drug sensitivity data published by the Wellcome Trust Sanger Institute (http://www.cancerrxgene.org/translation/Drug). We observed a trend towards sensitivity to cytarabine in the initial data sets, stratified by mutations in MSH2 vs wild-type (IC50 $0.067 \mu \mathrm{M} v s$ $0.788 \mu \mathrm{M}$ ) and mutations in MSH6 vs wild-type (IC50 $0.002 \mu \mathrm{M} v s$ $0.788 \mu \mathrm{M})$, with no clear association present with mutations in MLH1 vs wild-type $(0.682 \mu \mathrm{M} v s \quad 0.780 \mu \mathrm{M})$. Given that in the clinical setting the majority of MLH1 loss results from epigenetic modifications rather that the presence of mutations, we then stratified the IC50 data for cytarabine by MSI status, as MSI is present in the majority of dMMR tumours (Supplementary Table 2). We observed a statistically significant difference between 

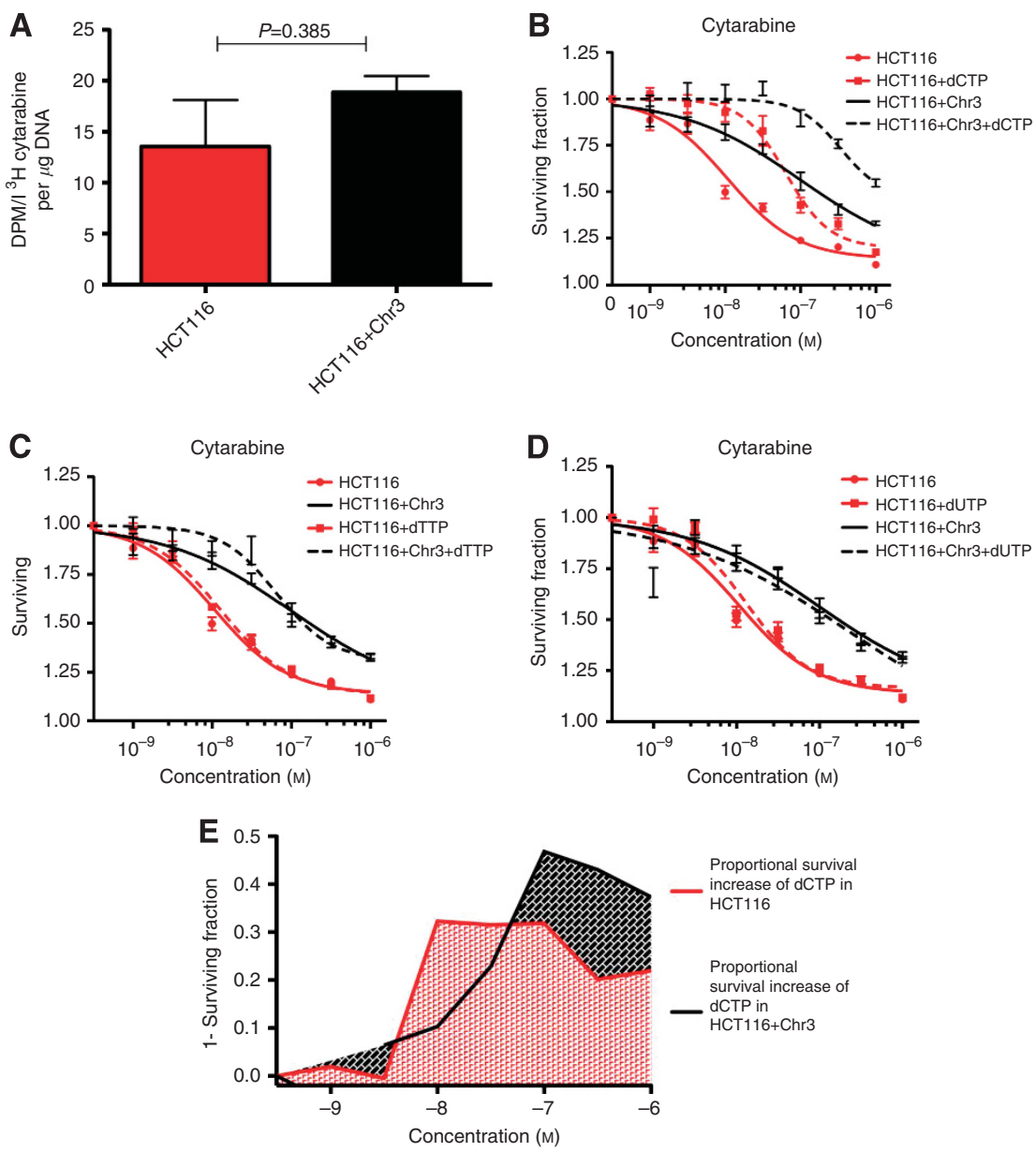

Figure 4. Cytarabine is not incorporated into DNA more in MLH1-deficient cells compared with MLH1-proficient cells. (A) Bar graph of incorporation of ${ }^{3} \mathrm{H}$-cytarabine into HCT116 and HCT116 + Chr3 cell lines. Cells were treated with cytarabine mixed with ${ }^{3} \mathrm{H}$-cytarabine for $72 \mathrm{~h}$. Genomic DNA was extracted and quantified. Levels of ${ }^{3} \mathrm{H}$-cytarabine per mg of DNA are shown $(P=0.3845$ (two-tailed t-test)). The experiment was performed in duplicate. (B-D) Survival curves of cytarabine exposure in the presence or absence of $10 \mathrm{~mm}$ dCTP, dTTP, or dUTP. Cellular viability was quantified after $72 \mathrm{~h}$ using the CellTiter-Glo assay, and SF calculated. (E) The incremental change in SF when HCT116 or HCT116 + Chr3 cells were exposed to cytarabine, with the addition of dCTP, when compared with cytarabine exposure alone (calculated from B).

cancer cells exhibiting MSI and those exhibiting microsatellite stability $(P=0.0388)$ (Figure $2 \mathrm{C}$ ). Given the relevance of dMMR to CRC in particular, we assessed the data arising from CRC cell lines alone (waterfall plot in Figure 2D), and also observed a statistically significant difference in cytarabine sensitivity dependent on MSI status across this smaller panel of cell lines $(P=0.0474)$. Taken together, these data suggest that the presence of dMMR/MSI may be one important modifier of sensitivity to cytarabine, although multiple other genetic and epigenetic factors are also important particularly given the multiple mechanisms of action of this drug (discussed below).

Given that MMR deficiency causes a mutator phenotype, it was still possible that genetic drift between isogenic clones could explain the phenotype we observed. We directly addressed this by inducing MLH1 deficiency in two isogenic systems using an orthogonal, siRNA approach. We transfected the MLH1-proficient cell line used in the screen, HCT116 $+\mathrm{Chr3}$, with non-targeting siRNA ( on cytarabine response (Supplementary Figure 2). We noticed a partial sensitization to cytarabine on MLH1 suppression. Notably, others have reported that adequate levels of MLH1 for MMR function in HCT116+Chr3 may persist even after loss of expression of MLH1 by western blot (Chauhan et al, 2000), We, therefore, selected the MLH1 isogenic system with the highest siRNA transfection rate, the A2780cp70 a2 (MLH1-deficient) and
A2780cp70 e1 (MLH1-proficient) ovarian cancer cells (Plumb et al, 2006), and performed the same experiment (Figure 2E(i)). While non-targeting siRNA did not ostensibly alter the response to cytarabine in either clone, MLH1 silencing in the previously MLH1-proficient el line (immunoblot in Figure 2E(ii)) did result in cytarabine sensitivity. Taken together, these results demonstrate that cytarabine sensitivity could be induced by MLH1 silencing and that mutations secondary to MMR deficiency were unlikely to explain the effects observed.

Other cytosine-based nucleoside analogues exhibit MMRdeficient selectivity. To begin to address the basis of the cytarabine selectivity, we performed cell viability assays to assess whether other cytosine-based nucleoside analogues might exhibit the same MLH1-deficient selective phenotype. Both cyclocytidine and azacytidine caused an MLH1 selective effect in isogenic CRC cells (Supplementary Figures 3a and b).

The cytarabine response of MLH1-deficient cancer cells is associated with increased apoptosis and a DNA damage response. As cytarabine is known to have multiple mechanisms of action, many of which are not well elucidated (Kufe et al, 1985; Grant, 1998), we next assessed the modalities of cell death and DNA damage associated with exposure to cytarabine in these cells. Cytarabine treatment has been associated with the induction of apoptosis in multiple models of cancer (Backway et al, 1997). To 

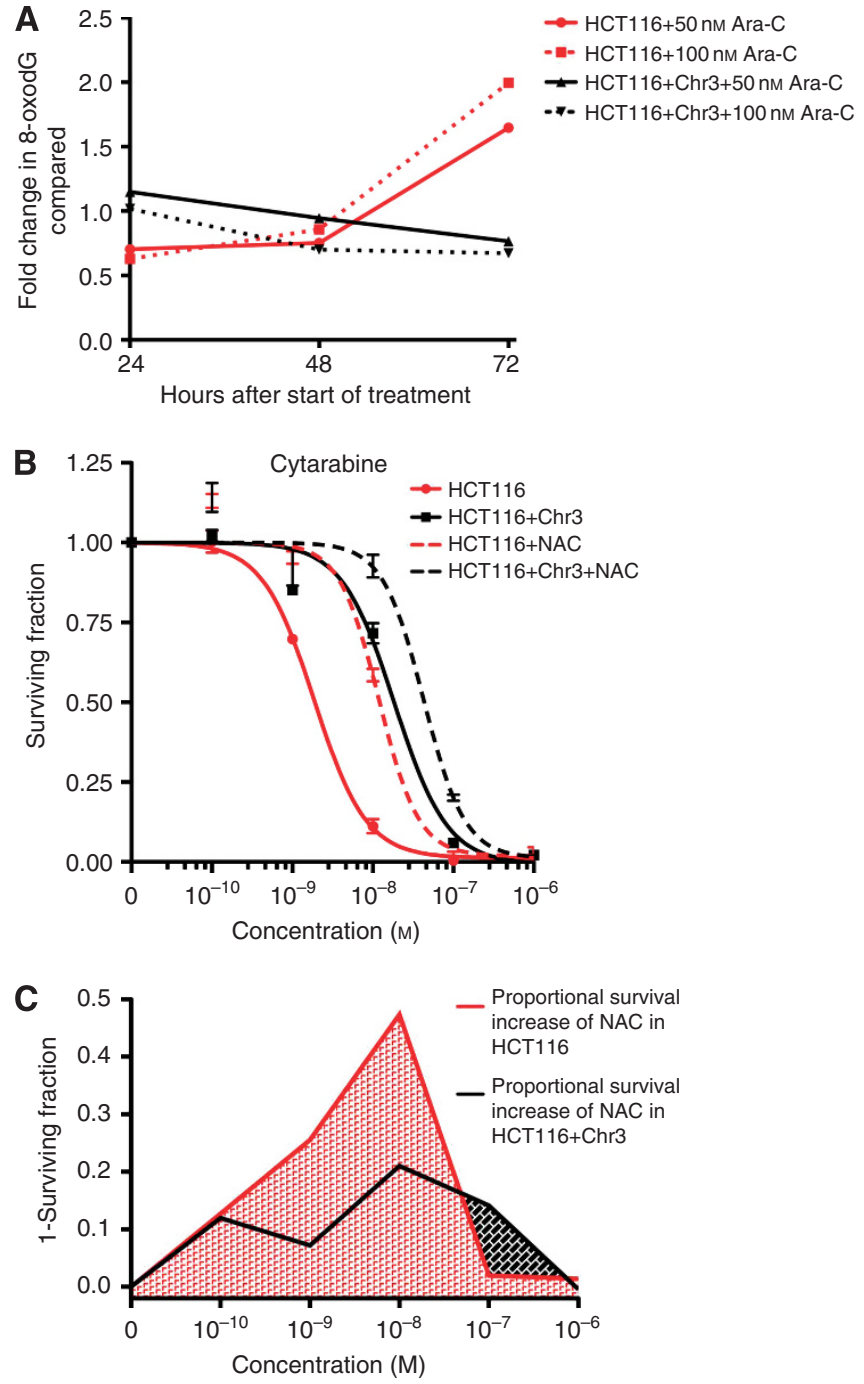

Figure 5. Cytarabine treatment in MMR-deficient cells is associated with an increase in oxidative DNA damage, and can be reversed by antioxidants. (A) Results of an ELISA assay to quantify levels of 8-oxodG in HCT116 and HCT116 + Chr3 following treatment with cytarabine. Cells were plated and exposed to cytarabine continuously $24 \mathrm{~h}$ later. DNA was extracted from whole-cell lysates after 24,48 , and $72 \mathrm{~h}$, and quantified; assay loading was normalised to total DNA concentration. Data from one representative experiment, performed in duplicate and analysed according to the manufacturer's protocol. 8-oxodG was quantified against a standard curve. (B) Clonogenic assay of the effects of NAC on cytotoxicity observed with cytarabine treatment. HCT116

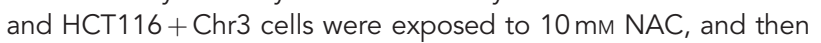
cytarabine or control for $24 \mathrm{~h}$. Cells were maintained in growth media until the end of the experiment (10-12 days). (C) Proportional increment in SF with the addition of NAC to cytarabine in HCT116 and HCT116+ Chr3 cells (calculated from B).

assess whether apoptosis was induced by cytarabine in these dMMR models, we extracted cell lysates from MLH1-deficient and proficient cells exposed to cytarabine/ ara-c for $72 \mathrm{~h}$, and quantified levels of cleaved PARP, produced as a consequence of caspase activation by immunoblotting. A significant concentration-dependent increase in PARP cleavage was observed in MLH1-deficient cells when compared with MLH1-proficient comparators at nanomolar concentrations (Figure 3A). Cytarabine is known to act as an inhibitor of DNA synthesis, competing with dCTP (Grant, 1998). Following conversion to its triphosphate form and incorporation into DNA, cytarabine triggers stalling of DNA replication forks, interfering with chain elongation and acting as a chain terminator. Cytarabine exposure has not been associated with potentially lethal double-strand DNA breaks (DSBs) in the manner of some cytotoxic agents (Grant, 1998; Pommier, 2006). We, therefore, determined the ability of cytarabine to induce a surrogate marker of replication fork stalling/DSB formation, histone $\mathrm{H} 2 \mathrm{AX}$ phosphorylation $(\gamma \mathrm{H} 2 \mathrm{AX})$ (Bonner et al, 2008). $\mathrm{H} 2 \mathrm{AX}$ is specifically phosphorylated on a C-terminal serine residue in response to DSBs and other types of DNA damage, including the stalling of DNA replication forks (Bonner et al, 2008). $\gamma \mathrm{H} 2 \mathrm{AX}$ levels were induced by cytarabine, and were significantly more elevated in MLH1-deficient HCT116 cells than MLH1-proficient HCT116 + Chr3 cells. The effect of cytarabine on $\gamma \mathrm{H} 2 \mathrm{AX}$ was concentration dependent and could be elicited at nanomolar concentrations, previously observed to be MLH1-deficient selective (Figures $3 \mathrm{~A}$ and $1 \mathrm{C}$ ). As $\mathrm{H} 2 \mathrm{AX}$ can also be phosphorylated in response to apoptosis, we also assessed nuclear $\gamma \mathrm{H} 2 \mathrm{AX}$ focus formation using immunofluorescence, which is relatively specific to DSB formation and the formation of stalled replication forks (Bonner et al, 2008). Cytarabine exposure additionally caused a significant elevation in the percentage of $\gamma \mathrm{H} 2 \mathrm{AX}$-positive cells in MLH1-deficient cells compared with MLH1 proficient (Figures 3B and $\mathrm{C}$ and Supplementary Figure 4). Collectively, these data suggested the MMR-deficient selectivity associated with cytarabine was associated with induction of apoptosis and activation of the DNA damage response.

Investigation of the potential mechanism of MMR selectivity. Cytarabine is initially converted to a monophosphate form, and then to a diphosphate and triphosphate (cytarabine triphosphate; ara-CTP), followed by incorporation into DNA. The degree of ara-CTP incorporation correlates with the degree of resulting cytotoxicity (Grant, 1998). As we had observed induction of the DNA damage response, we next assessed whether the MMRdeficient selective effect of cytarabine could be explained by differential incorporation into DNA. We determined the relative incorporation of ${ }^{3} \mathrm{H}$-cytarabine into DNA in MLH1-deficient and proficient cells, and did not observe a significantly differing rate between the two models (Figure 4A). We also assessed the effects of exogenous nucleosides (Figure $4 \mathrm{~B}-\mathrm{D}$ ). Although the presence of dCTP did ameliorate the inhibitory effects of cytarabine, it did so in equal measure in both cell lines (Figure 4E). Taken together, these data suggested that the increased sensitivity of MLH1deficient cell lines could not be explained by the differential incorporation of cytarabine into DNA.

Cytarabine and other nucleoside analogues are also known to inhibit the DNA polymerase POLA, with a lesser inhibition of $P O L B$, albeit weakly at micromolar concentrations (Grant, 1998; Wills et al, 2000). In view of these data, and data from our laboratory suggesting synthetic lethal relationships between MLH1 and POLG, and MSH2 and POLB (Martin et al, 2010), we tested the MLH1 selective effects of inhibition of POLA or its regulatory subunit POLA2 using siRNA in HCT116 and HCT116+Chr3 cells. We did not observe a significant difference in response (Supplementary Figure 5). The absence of MLH1-POLA synthetic lethality coupled with the concentrations at which this phenotype was observed, suggested that the MMR selective effect of cytarabine was less likely to be explained by an effect of cytarabine on POLA.

The MLH1-deficient selective effect of cytarabine can be abrogated by antioxidants and is associated with increased oxidatively damaged DNA. Our previous studies (Martin et al, 2009, 2010, 2011), together with those of others (Macpherson et al, 2005), have highlighted the dMMR selective potential of drugs and other cellular perturbations that induce intracellular oxidative stress. One hypothesis is that these observations can be explained by the role of MMR in the repair of oxidatively damaged DNA, and a relative failure of these processes in MMR-deficient tumour cells 


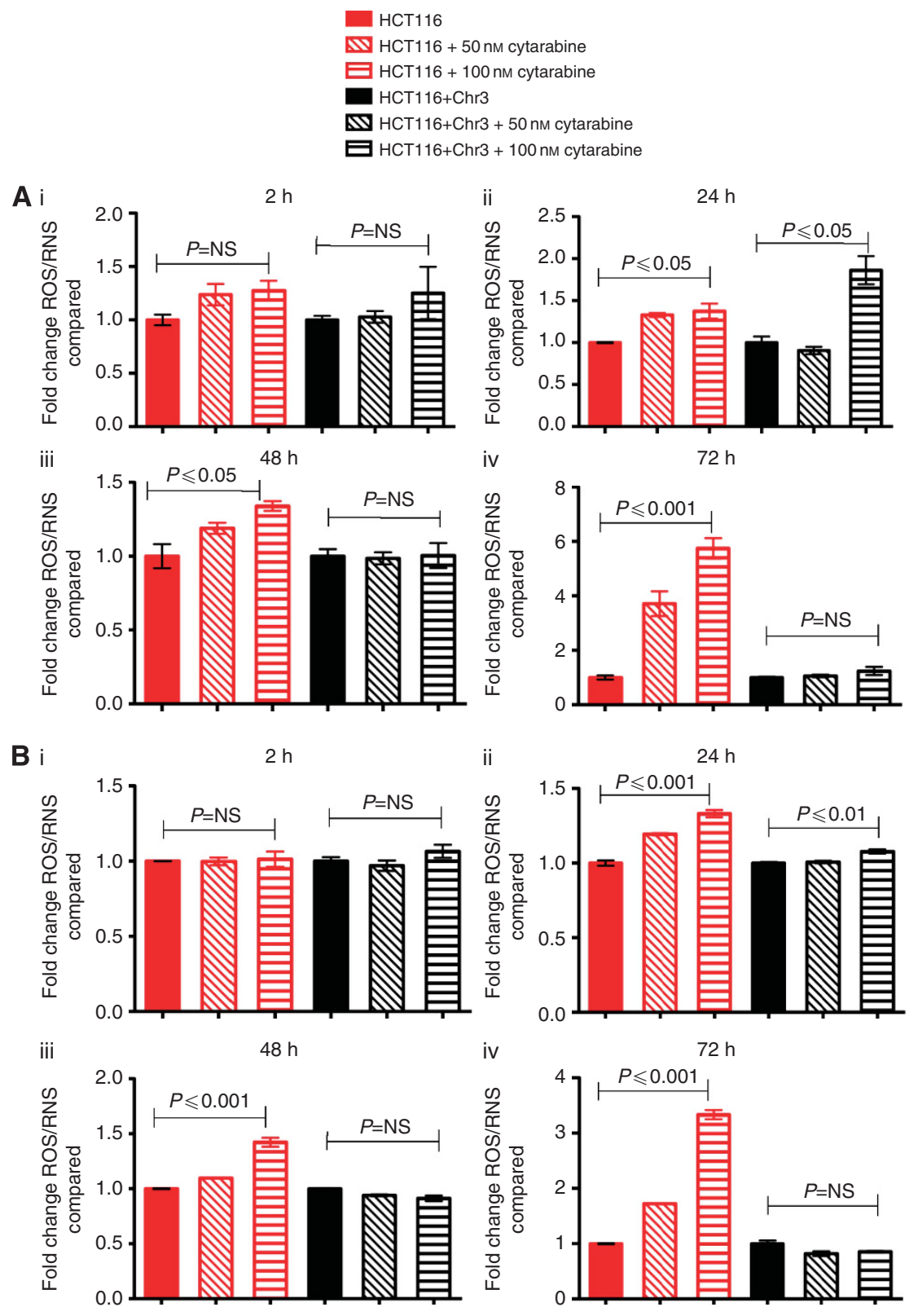

Figure 6. Cytarabine treatment in MMR-deficient cells is associated with an increase in ROS in a dose-dependent manner. Quantification of ROS in HCT116 and HCT116 + Chr3 cells exposed to cytarabine or control for different durations. Samples were taken from homogenised cells (A) and growth media (B). The resulting fluorescent signal was quantified using a 96-well plate reader. Assays were performed in triplicate. Error bars represent the s.e.m. Statistical values were obtained using a two-tailed t-test.

(Martin et al, 2010). As it has previously been demonstrated that cellular exposure to cytarabine was associated with increased oxidative stress (Geller et al, 2001), and that oxidative stress can result in DNA damage, with excess levels potentially resulting in formation of $\gamma \mathrm{H} 2 \mathrm{AX}$ and cytotoxicity (Trachootham et al, 2009), we reasoned that oxidative stress and oxidatively damaged DNA might be responsible for the MMR-deficient selectivity of cytarabine.

We exposed the MLH1-deficient and proficient cells to cytarabine, and measured levels of 8-oxodG in DNA, a wellvalidated marker of oxidatively damaged DNA (Evans et al, 2010; Cadet et al, 2012) using an ELISA assay, as previously described (Martin et al, 2010). Cytarabine exposure resulted in an increase in levels of 8-oxodG in MLH1-deficient cells after $72 \mathrm{~h}$, which was not observed in MLH1-proficient cells (Figure 5A). Given the role of MLH1 in the repair of mitochondrial oxidative damage (Martin et al, 2010), we examined 8-oxodG levels in mitochondrial DNA, and observed a particularly marked increase in the levels of mitochondrial 8-oxodG in MLH1-deficient cells when compared with nuclear DNA (Supplementary Figure 6).

If cytarabine caused MLH1 selective effects via an oxidative stress-mediated mechanism, then we reasoned that then antioxidant compounds might modify the impact of cytarabine in MLH1-deficient cells. We exposed HCT116 and HCT116+ Chr3 cells to media containing $10 \mathrm{~mm} \mathrm{~N}$-acetyl-L-cysteineNAC, a scavenger of ROS (Raj et al, 2011), and cytarabine. We found that NAC almost totally abrogated the excess cytotoxicity of cytarabine observed in MLH1-deficient cells (Figure 5B). Exposure to NAC reduced the difference in the size of the therapeutic window observed between the cell lines, suggesting that a prooxidant mechanism might be important in mediating MLH1 selectivity (Figure 5C). 
The MLH1-deficient selective effect of cytarabine is associated with presence of increased levels of ROS and collapse of the mitochondrial membrane potential. As cytarabine has been associated with the production of ROS (Geller et al, 2001), we investigated whether cytarabine induced ROS at the concentrations eliciting MMR selectivity over a time course. We observed modestly increased levels of ROS in MLH1-deficient cells exposed to cytarabine after $2 \mathrm{~h}$ of treatment. Reactive oxygen species levels became further elevated over time, in a dose-dependent manner, in both cells and later in culture supernatant samples (Figure 6A and B). Notably, whilst some initial increase in ROS was observed in MLH1-proficient cells (Figure 6A(i) and (ii)), this was not observed at later time points (Figure 6A(iii) and (iv)).

Finally, given previous data suggesting that the apoptosis associated with cytarabine treatment occurred following collapse of the mitochondrial membrane potential $(\Delta \Psi \mathrm{m})$ (Backway et al, 1997), we quantified the reduction of $\Delta \Psi \mathrm{m}$ with Mitotracker red (Molecular Probes, Invitrogen, Life Technologies) after cytarabine treatment over a time course, along with Annexin V and DAPI staining to further characterise cell populations for cellular viability and apoptosis. Mitotracker red is a cell permeable dye selective for mitochondria, with mitochondrial accumulation only taking place in the presence of an adequate mitochondrial membrane potential. We observed an increase in the proportion of Mitotracker rednegative cells (both Annexin V negative cells, and Annexin V positive, apoptotic cells), dependent on length of exposure to drug and concentration, and greater in the MLH1-deficient cells (Supplementary Figures $7 \mathrm{a}-\mathrm{d}$ ). Taken together, our data suggested that cytarabine caused increased cytotoxicity in MLH1-deficient CRC cells due to its ability to generate instability of the $\Delta \Psi \mathrm{m}$ and generate ROS. Firstly, this could lead directly to apoptosis, as excess levels of ROS were not controlled and gradually accumulated, with ROS levels presumably reaching a critical threshold because of high pre-existing endogenous levels in MLH1-deficient cells (Trachootham et al, 2009). Secondly, this could lead to suboptimal repair of oxidatively damaged DNA. The presence of higher levels of oxidatively damaged DNA likely leads to an increased number of stalled replication forks, collapsed replication forks, and ultimately the potential for the formation of lethal DSBs and an apoptotic response (Supplementary Figure 8).

\section{DISCUSSION}

The findings described here demonstrate the potential value of comprehensive drug screens in re-purposing established drugs for the treatment of particular molecular subsets of cancer. We demonstrated through a screen of isogenic MLH1-deficient and proficient cancer cell lines that cytosine-based nucleoside analogues were selectively cytotoxic to MMR-deficient cells, most likely as a result of their ability to alter the $\Delta \Psi \mathrm{m}$ and generate ROS. Our data suggest that treatment of CRC cells with low concentrations of cytarabine results in early production of ROS and destabilisation of the mitochondrial membrane potential. In the absence of MLH1 or $\mathrm{MSH} 2$, apoptosis may result both from uncontrolled ROS levels due to an inadequate or overwhelmed antioxidant response, and also from an inability to repair oxidatively damaged DNA leading to an increase in potentially lethal DSB formation and apoptosis. Notably, others have reported that the ability of cytarabine to cause apoptosis in cultured post-mitotic neurons, which do not express POLA, occurs at low concentrations that do not result in nuclear DNA incorporation, and is mediated by oxidative stress and mitochondrial permeability transition (Geller et al, 2001; Xue et al, 2002).

Our initial observation that MMR-deficient cells are sensitive to nucleoside analogues is supported by some other work. Fordham et al (2011) observed that an MSH6-deficient lymphoma cell line model exhibited increased sensitivity to cytarabine, along with a leukaemia cell line in which expression was inhibited using short hairpin RNA. Takahashi et al (2005) reported that MMR-deficient cells were sensitised to DNA polymerase reaction inhibitors, including cytarabine. However, in view of the fact that we observed no differential selectivity when we specifically inhibited POLA, and that we observed sensitization at one hundredth of the concentration that is required for significant inhibition of DNA polymerases (Furth and Cohen, 1968; Grant, 1998), a causal relationship appears less likely from our data. Our data in four isogenic models together with analysis of publicly available data sets assessing multiple non-isogenic models demonstrates that MMR selectivity of cytarabine in epithelial and haematological cancer cells is a relatively robust effect, and provides greater impetus that cytarabine should be assessed clinically in patients with MMRdeficient malignancies.

In order to take forward these observations into the clinical setting, robust biomarkers are required to ensure that the target effect is achieved in vivo; in this case, that oxidatively damaged DNA is generated. The 8-oxodG ELISA assay has been in widespread use in research outside the field of oncology in the investigation of oxidative stress in Parkinson's disease and diabetes. The efforts of ESCULA (European Standard Committee of Urinary (DNA) Lesion Analysis) among others (Evans et al, 2010) will be essential to the further development of reliable assays for clinical use.

In the clinic, low-dose cytarabine treatment results in plasma drug levels of 42-64 nmoll $^{-1}$ (Kufe et al, 1985). This has been reported as sufficient to result in many of the cytarabine-associated phenotypes, including the delayed replication of human leukaemic cells in vitro, and oxidative stress. Provided that these in vitro results can be replicated in vivo, a clinical trial of low-dose cytarabine, or a cytarabine-based combination, in the dMMR subset of epithelial cancers most likely to respond to it represents an intriguing possibility.

\section{ACKNOWLEDGEMENTS}

We acknowledge National Health Service funding to the National Institute for Health Research Royal Marsden Hospital/Institute of Cancer Research Biomedical Research Centre. Madeleine Hewish was in receipt of a Clinical Research Training Fellowship from the Medical Research Council. This work was also funded by a programme grant from Cancer Research UK (C347/A8363) (Alan Ashworth) and annual grants from Breakthrough Breast Cancer (BC 08/09, BC 09/10 (Alan Ashworth) and CTR-Q3Y-Y1 (Clare Isacke)).

\section{CONFLICT OF INTEREST}

Professor Ashworth and Dr Lord are co-investigators on patents held with Kudos-Astra Zeneca for the development of PARP inhibitors and may also benefit through the Institute of Cancer Research 'Rewards to Inventors' scheme. Professor Ashworth, Dr Lord, and Dr Martin have a patent pending on the use of methotrexate in mismatch repair-deficient cancers. Professor Ashworth has received honoraria from Roche, Merck Serono, and Clovis Oncology, and is a member of the scientific advisory board of GSK. The remaining authors declare no conflict of interest.

\section{REFERENCES}

Ashworth A, Lord CJ, Reis-Filho JS (2011) Genetic interactions in cancer progression and treatment. Cell 145: 30-38.

Backway KL, McCulloch EA, Chow S, Hedley DW (1997) Relationships between the mitochondrial permeability transition and oxidative stress during ara-C toxicity. Cancer Res 57: 2446-2451. 
Bonner WM, Redon CE, Dickey JS, Nakamura AJ, Sedelnikova OA, Solier S, Pommier Y (2008) GammaH2AX and cancer. Nat Rev Cancer 8: 957-967.

Brown JM, Attardi LD (2005) The role of apoptosis in cancer development and treatment response. Nat Rev Cancer 5: 231-237.

Cadet J, Loft S, Olinski R, Evans MD, Bialkowski K, Richard Wagner J, Dedon PC, Moller P, Greenberg MM, Cooke MS (2012) Biologically relevant oxidants and terminology, classification and nomenclature of oxidatively generated damage to nucleobases and 2-deoxyribose in nucleic acids. Free Radic Res 46: 367-381.

Chauhan DP, Yang Q, Carethers JM, Marra G, Chang CL, Chamberlain SM, Boland CR (2000) Antisense inhibition of hMLH1 is not sufficient for loss of DNA mismatch repair function in the HCT116 + chromosome 3 cell line. Clin Cancer Res 6: 3827-3831.

Evans MD, Olinski R, Loft S, Cooke MS (2010) Toward consensus in the analysis of urinary 8-oxo-7,8-dihydro-2'-deoxyguanosine as a noninvasive biomarker of oxidative stress. FASEB J 24: 1249-1260.

Fordham SE, Matheson EC, Scott K, Irving JA, Allan JM (2011) DNA mismatch repair status affects cellular response to Ara-C and other anti-leukemic nucleoside analogs. Leukemia 25: 1046-1049.

Furth JJ, Cohen SS (1968) Inhibition of mammalian DNA polymerase by the $5^{\prime}$-triphosphate of 1-beta-d-arabinofuranosylcytosine and the $5^{\prime}$-triphosphate of 9-beta-d-arabinofuranoxyladenine. Cancer Res 28: 2061-2067.

Geller HM, Cheng KY, Goldsmith NK, Romero AA, Zhang AL, Morris EJ, Grandison L (2001) Oxidative stress mediates neuronal DNA damage and apoptosis in response to cytosine arabinoside. J Neurochem 78: $265-275$.

Grant S (1998) Ara-C: cellular and molecular pharmacology. Adv Cancer Res 72: 197-233.

Hewish M, Lord CJ, Martin SA, Cunningham D, Ashworth A (2010) Mismatch repair deficient colorectal cancer in the era of personalized treatment. Nat Rev Clin Oncol 7: 197-208.

Jiricny J (2006) The multifaceted mismatch-repair system. Nat Rev Mol Cell Biol 7: 335-346.

Koi M, Umar A, Chauhan DP, Cherian SP, Carethers JM, Kunkel TA, Boland CR (1994) Human chromosome 3 corrects mismatch repair deficiency and microsatellite instability and reduces $\mathrm{N}$-methyl- $\mathrm{N}^{\prime}$-nitro- $\mathrm{N}$ nitrosoguanidine tolerance in colon tumor cells with homozygous hMLH1 mutation. Cancer Res 54: 4308-4312.

Kufe DW, Griffin JD, Spriggs DR (1985) Cellular and clinical pharmacology of low-dose ara-C. Semin Oncol 12: 200-207.

Laux I, Nel A (2001) Evidence that oxidative stress-induced apoptosis by menadione involves Fas-dependent and Fas-independent pathways. Clin Immunol 101: 335-344.

Lynch HT, Lynch JF, Lynch PM, Attard T (2008) Hereditary colorectal cancer syndromes: molecular genetics, genetic counseling, diagnosis and management. Fam Cancer 7: 27-39.

Macpherson P, Barone F, Maga G, Mazzei F, Karran P, Bignami M (2005) 8-oxoguanine incorporation into DNA repeats in vitro and mismatch recognition by MutSalpha. Nucleic Acids Res 33: 5094-5105.

Maeng CH, Lu S, Park SH, Hummelen PV, MacConaill LE, Kim KM, Lee J (2012) Screening for somatic mutations in esophageal squamous cell carcinoma using OncoMap 4.0. J Clin Oncol 30: 4S (abstract 27).

Martin SA, Hewish M, Sims D, Lord CJ, Ashworth A (2011) Parallel highthroughput RNA interference screens identify PINK1 as a potential therapeutic target for the treatment of DNA mismatch repair-deficient cancers. Cancer Res 71: 1836-1848.
Martin SA, McCabe N, Mullarkey M, Cummins R, Burgess DJ, Nakabeppu Y, Oka S, Kay E, Lord CJ, Ashworth A (2010) DNA polymerases as potential therapeutic targets for cancers deficient in the DNA mismatch repair proteins MSH2 or MLH1. Cancer Cell 17: 235-248.

Martin SA, McCarthy A, Barber LJ, Burgess DJ, Parry S, Lord CJ, Ashworth A (2009) Methotrexate induces oxidative DNA damage and is selectively lethal to tumour cells with defects in the DNA mismatch repair gene MSH2. EMBO Mol Med 1: 323-337.

Plumb JA, Aherne GW, Lee D, Westwood N, O’Brien V, McDonald E, Brown $\mathrm{R}$ (2006) MMR201: A novel small molecule that selectively inhibits growth of MLH1 deficient tumor cells. Abstract presented at: AACR Annual Meeting 2006. (abstract 2435).

Pommier Y (2006) Topoisomerase I inhibitors: camptothecins and beyond. Nat Rev Cancer 6: 789-802.

Raj L, Ide T, Gurkar AU, Foley M, Schenone M, Li X, Tolliday NJ, Golub TR, Carr SA, Shamji AF, Stern AM, Mandinova A, Schreiber SL, Lee SW (2011) Selective killing of cancer cells by a small molecule targeting the stress response to ROS. Nature 475: 231-234.

Takahashi T, Min Z, Uchida I, Arita M, Watanabe Y, Koi M, Hemmi H (2005) Hypersensitivity in DNA mismatch repair-deficient colon carcinoma cells to DNA polymerase reaction inhibitors. Cancer Lett 220: 85-93.

Tejpar S, Bertagnolli M, Bosman F, Lenz HJ, Garraway L, Waldman F, Warren R, Bild A, Collins-Brennan D, Hahn H, Harkin DP, Kennedy R, Ilyas M, Morreau H, Proutski V, Swanton C, Tomlinson I, Delorenzi M, Fiocca R, Van Cutsem E, Roth A (2010) Prognostic and predictive biomarkers in resected colon cancer: current status and future perspectives for integrating genomics into biomarker discovery. Oncologist 15: 390-404.

Trachootham D, Alexandre J, Huang P (2009) Targeting cancer cells by ROS-mediated mechanisms: a radical therapeutic approach? Nat Rev Drug Discov 8: 579-591.

Tutt A, Robson M, Garber JE, Domchek SM, Audeh MW, Weitzel JN, Friedlander M, Arun B, Loman N, Schmutzler RK, Wardley A, Mitchell G, Earl H, Wickens M, Carmichael J (2010) Oral poly(ADP-ribose) polymerase inhibitor olaparib in patients with BRCA1 or BRCA2 mutations and advanced breast cancer: a proof-of-concept trial. Lancet 376: $235-244$

Watanabe Y, Haugen-Strano A, Umar A, Yamada K, Hemmi H, Kikuchi Y, Takano S, Shibata Y, Barrett JC, Kunkel TA, Koi M (2000) Complementation of an hMSH2 defect in human colorectal carcinoma cells by human chromosome 2 transfer. Mol Carcinog 29: $37-49$.

Weiss MB, Vitolo MI, Baerenfaller K, Marra G, Park BH, Bachman KE (2007) Persistent mismatch repair deficiency following targeted correction of hMLH1. Cancer Gene Ther 14: 98-104.

Wills PW, Hickey R, Malkas L (2000) Ara-C differentially affects multiprotein forms of human cell DNA polymerase. Cancer Chemother Pharmacol 46: 193-203.

Xue L, Borutaite V, Tolkovsky AM (2002) Inhibition of mitochondrial permeability transition and release of cytochrome $\mathrm{c}$ by anti-apoptotic nucleoside analogues. Biochem Pharmacol 64: 441-449.

This work is published under the standard license to publish agreement. After 12 months the work will become freely available and the license terms will switch to a Creative Commons AttributionNonCommercial-Share Alike 3.0 Unported License.

Supplementary Information accompanies this paper on British Journal of Cancer website (http://www.nature.com/bjc) 